\title{
Supporting Information: Structure and dynamics of interacting nanoparticles in semidilute polymer solutions
}

\author{
Ryan Poling-Skutvik, ${ }^{\dagger}$ Katrina Irene S. Mongcopa,${ }^{\dagger}$ Antonio Faraone, ${ }^{\ddagger}$ Suresh \\ Narayanan, $₫$ Jacinta C. Conrad, ${ }^{*, \dagger}$ and Ramanan Krishnamoorti ${ }^{* \dagger, \S}$ \\ $\dagger$ Department of Chemical and Biomolecular Engineering, University of Houston, Houston, \\ $T X 77204$ \\ $\ddagger$ National Institute of Standards and Technology Center for Neutron Research, \\ Gaithersburg, MD 20899 \\ \Advanced Photon Source, Argonne National Laboratory, Argonne, IL 60439 \\ $\S$ Department of Chemistry, University of Houston, Houston, TX 77204 \\ E-mail: jcconrad@uh.edu; ramanan@uh.edu
}

\section{Intrinsic viscosity and dynamic light scattering}

A stock solution of polystyrene is prepared from $0.159 \mathrm{~g}$ of $706 \mathrm{kDa}$ polystyrene and 11.724 $\mathrm{g}$ of 2-butanone for a final concentration of $1.019 \mathrm{c} / \mathrm{c}^{*}$. The stock solution is rolled for 2 days to homogenize. From the stock solution, dilute polystyrene solutions are prepared by mixing appropriate amounts of the stock solution and additional 2-butanone. The dilute solutions are passed through a capillary viscometer (Cannon 0C) suspended in a water bath at $25^{\circ} \mathrm{C}$. The capillary viscometer produces large shear rates of approximately $200 \mathrm{~s}^{-1}$ to $300 \mathrm{~s}^{-1}$. The measured viscosities are shown in Fig. S1. The intrinsic viscosity $[\eta]=(0.104 \pm 0.006) \mathrm{L} / \mathrm{g}$ is 
calculated from the change in bulk viscosity $\eta$ as a function of polymer concentration through $\eta=\eta_{0}(1+2.5[\eta] c) .^{\dagger}$ The overlap concentration is calculated to be $c^{*}=1 /[\eta]=(9.7 \pm 0.6)$ $\mathrm{g} / \mathrm{L}$, in good agreement with literature values for polystyrene in 2-butanone. ${ }^{\text {S1-S3 }}$

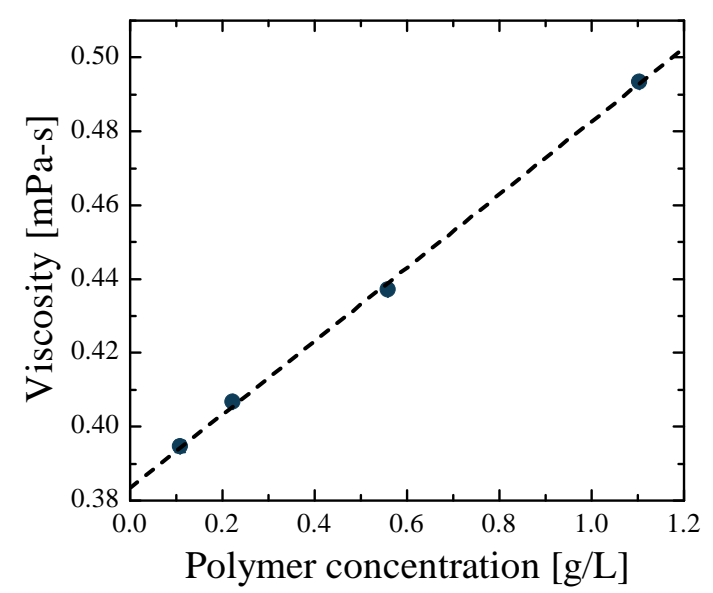

Figure S1: Viscosity $\eta$ of $706 \mathrm{kDa}$ polystyrene solutions at $25^{\circ} \mathrm{C}$ as measured in a capillary viscometer at estimated shear rates of approximately $200 \mathrm{~s}^{-1}$ to $300 \mathrm{~s}^{-1}$. The dashed line represents a linear fit with a slope of $(0.099 \pm 0.002) \mathrm{mPa} \cdot \mathrm{s} /(\mathrm{g} / \mathrm{L})$ and an intercept of $(0.38$ $\pm 0.03) \mathrm{mPa} \cdot \mathrm{s}$.

To the same solutions, we add $10 \mu \mathrm{L}$ of the particle stock solution from Nissan Chemical, which was filtered through a $0.2 \mu \mathrm{m}$ PTFE filter. The final particle volume fraction is 8 $\times 10^{-5}$. We use these solutions for dynamic light scattering measurements to determine the particle radius in the presence of polystyrene. The DLS curves are fit with a cumulant model to extract out the relaxation rate $\Gamma=D Q^{2}$, where $D$ is the diffusivity of the particle. We calculate the radius of the particles from the diffusivity measurements using the StokesEinstein equation and the viscosities in Fig. S1.

\section{Detailed sample concentrations}

Samples are prepared according to the procedure in the main body of the text. For x-ray scattering and USANS experiments, the precise sample compositions are detailed in Table SI.

\footnotetext{
${ }^{\dagger}$ Throughout this manuscript, error bars and uncertainties represent one standard deviation.
} 
For neutron experiments, the silica particles are first vacuum dried at room temperature for 8 hours. Particles are then added to d5-2-butanone in the appropriate ratio and sonicated with a probe sonicator (Q500, QSonica) at $25 \%$ power in cycles of 5 seconds on and 55 seconds off for a total of one hour. The stock solution of particles in d5-2-butanone contains $1.003 \mathrm{~g}$ of dried silica particles with $2.342 \mathrm{~g}$ of d5-2-butanone. A small fraction of the solvent $(0.070 \mathrm{~g})$ evaporates during sonication and the final particle concentration is $30.6 \%$ by weight silica. The stock particle solution is then mixed with additional d5-2-butanone and polystyrene according to the values listed in Table SII.

Table SI: Sample compositions for protonated 2-butanone solutions. Particle stock solution (MEK-ST-L) contains $30 \%$ by weight silica particles in 2-butanone according to manufacturer.

\begin{tabular}{|c|c|c|c|c|l|}
\hline $\begin{array}{c}\text { Nominal } \\
\text { Concentrations }\end{array}$ & $\begin{array}{c}\text { Mass } \\
\text { PS }[\mathrm{g}]\end{array}$ & $\begin{array}{c}\text { Mass } \\
\text { 2-butanone }[\mathrm{g}]\end{array}$ & $\begin{array}{c}\text { Mass } \\
\text { MEK-ST-L [g] }\end{array}$ & PS [c/c*] & $\begin{array}{l}\text { Mass } \\
\text { percent } \\
\mathrm{SiO}_{2}\end{array}$ \\
\hline $5.5 \mathrm{c}^{*} \mathrm{PS} 1 \% \mathrm{SiO}_{2}$ & 0.070 & 0.897 & 0.036 & 5.33 & 1.09 \\
\hline $5.5 \mathrm{c}^{*} \mathrm{PS} 3 \% \mathrm{SiO}_{2}$ & 0.070 & 0.837 & 0.103 & 5.42 & 3.05 \\
\hline $5.5 \mathrm{c}^{*} \mathrm{PS} 10 \% \mathrm{SiO}_{2}$ & 0.069 & 0.599 & 0.331 & 5.61 & 9.94 \\
\hline $8 \mathrm{c}^{*} \mathrm{PS} 3 \% \mathrm{SiO}_{2}$ & 0.095 & 0.810 & 0.102 & 7.35 & 3.03 \\
\hline $12.5 \mathrm{c}^{*} \mathrm{PS} 1 \% \mathrm{SiO}_{2}$ & 0.155 & 0.804 & 0.038 & 12.1 & 1.16 \\
\hline $12.5 \mathrm{c}^{*} \mathrm{PS} 10 \% \mathrm{SiO}_{2}$ & 0.157 & 0.532 & 0.328 & 12.8 & 9.68 \\
\hline
\end{tabular}

Table SII: Sample compositions for partially deuterated d5-2-butanone solutions. Particle stock solution (d5-MEK-ST-L) contains $30.6 \%$ by weight silica particles in d5-2-butanone.

\begin{tabular}{|c|c|c|c|c|l|}
\hline $\begin{array}{c}\text { Nominal } \\
\text { Concentrations }\end{array}$ & $\begin{array}{c}\text { Mass } \\
\text { PS }[\mathrm{g}]\end{array}$ & $\begin{array}{c}\text { Mass } \\
\text { d5-2-butanone }[\mathrm{g}]\end{array}$ & $\begin{array}{c}\text { Mass } \\
\text { d5-MEK-ST-L }[\mathrm{g}]\end{array}$ & PS $\left[\mathrm{c} / \mathrm{c}^{*}\right]$ & $\begin{array}{l}\text { Mass } \\
\text { percent } \\
\mathrm{SiO}_{2}\end{array}$ \\
\hline $8 \mathrm{c}^{*} \mathrm{PS} 0 \% \mathrm{SiO}_{2}$ & 0.308 & 2.770 & 0 & 8.17 & 0 \\
\hline $8 \mathrm{c}^{*} \mathrm{PS} 1 \% \mathrm{SiO}_{2}$ & 0.311 & 2.678 & 0.110 & 8.24 & 1.06 \\
\hline $8 \mathrm{c}^{*} \mathrm{PS} 10 \% \mathrm{SiO}_{2}$ & 0.317 & 1.799 & 1.036 & 8.73 & 9.86 \\
\hline $16.5 \mathrm{c}^{*} \mathrm{PS} 1 \% \mathrm{SiO}_{2}$ & 0.628 & 2.414 & 0.146 & 16.4 & 1.38 \\
\hline $18.5 \mathrm{c}^{*} \mathrm{PS} 10 \% \mathrm{SiO}_{2}$ & 0.434 & 1.004 & 0.720 & 17.8 & 10.1 \\
\hline
\end{tabular}




\section{SANS and USANS comparison}

We collect both SANS and USANS data on the same solution to measure structure over length scales ranging from $1 \mathrm{~nm}$ to over 1 micron. Because the USANS data is collected using a slit geometry, the resulting scattering intensity is heavily smeared. After desmearing, the USANS data overlaps within experimental error with the SANS data (Fig. S2).

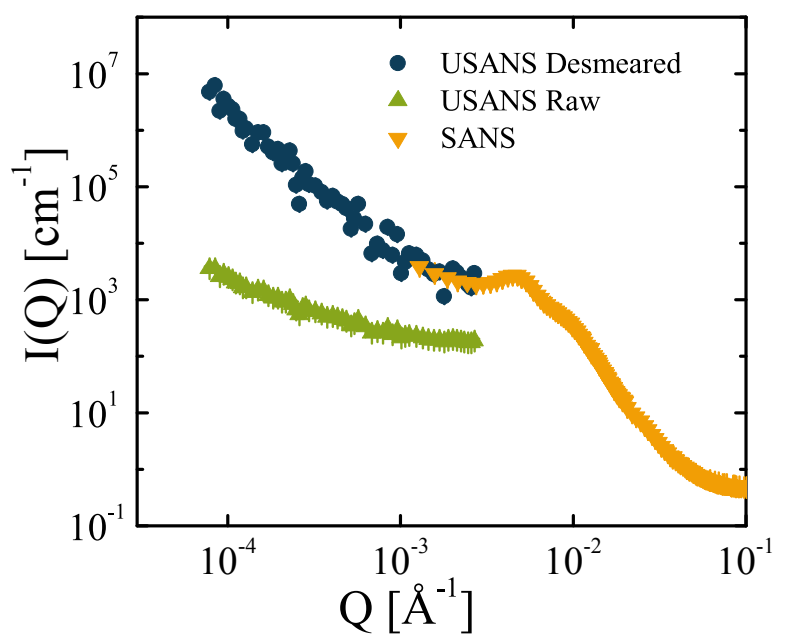

Figure S2: SANS and USANS scattering intensity for the $8 \mathrm{c}^{*} \mathrm{PS} 10 \% \mathrm{SiO}_{2}$ solution in protonated 2-butanone. USANS data was desmeared using the slit geometry of the beamline as described in the main text.

\section{Incoherent Scattering}

To analyze the SANS (SAXS) data, we first subtract the incoherent (background) data. We determine the incoherent (background) scattering intensity by linear fits to $I(Q) Q^{4}$ vs $Q^{4}$. The slope of the data for $Q \gtrsim 0.1 \AA^{-1}$ in Fig. S3 corresponds to the incoherent scattering intensity, which is subtracted from the total intensity to give $I_{\text {coh }}(Q)=I(Q)-I_{\text {incoh }}(Q)$. The same procedure is used to remove the incoherent (background) scattering from all the data presented in the main body of the text. 


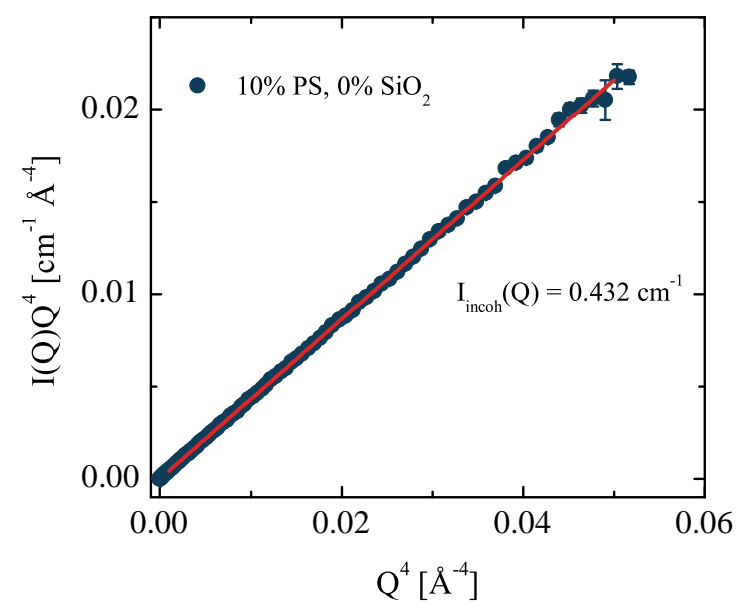

Figure S3: SANS data for the $8 \mathrm{c}^{*} \mathrm{PS} 0 \% \mathrm{SiO}_{2}$ solution in d5-2-butanone. Red line indicates a linear fit with a slope of $0.432 \mathrm{~cm}^{-1}$.

\section{$5 \quad$ Aggregation in concentrated solutions}

The structure of the particle dispersions in polymer solutions changes significantly once the polymer concentration $\mathrm{c} / \mathrm{c}^{*}>15$. For the solutions described in the main body of text nominal polymer concentrations of $5.5 \mathrm{c}^{*}, 8 \mathrm{c}^{*}$, and $12.5 \mathrm{c}^{*}$ - the particles are well dispersed. Above $15 \mathrm{c}^{*}$, the structure factor peak disappears and upturns in $I(Q)$ appear at low- $Q$, signifying aggregation of the particles (Fig. S4). To characterize the size of the aggregates, we fit the data on a Guinier plot (inset to Fig. S4), according to

$$
I(Q)=I_{0} \exp \left(-\left(R_{\mathrm{g}} Q\right)^{2} / 3\right)
$$

where $I_{0}$ represents the scattering intensity at low $Q$ and $R_{\mathrm{g}}$ represents the radius of gyration of the aggregates. The linearity of the data for both solutions at low $Q$ indicate that the aggregates have a finite size of $R_{\mathrm{g}}=(51 \pm 1) \mathrm{nm}$ and $(68 \pm 0.5) \mathrm{nm}$ for the $1 \%$ by weight and $10 \%$ by weight silica loadings, respectively. Using the fractal size, we fit $I(Q)$ to the sum of two spherical form factors according to $I(Q)=A P_{\text {ind }}(Q)+B P_{\text {agg }}(Q)$ where $A$ and 
$B$ are scaling factors,

$$
P_{\mathrm{i}}(Q)=\left[\frac{\sin \left(Q R_{\mathrm{i}}\right)-Q R_{\mathrm{i}} \cos \left(Q R_{\mathrm{i}}\right)}{\left(Q R_{\mathrm{i}}\right)^{3}}\right]^{2},
$$

and $R_{\mathrm{i}}$ is the radius of individual particles and aggregates, respectively. We set $R_{\text {ind }}=24$ $\mathrm{nm}$ and $R_{\mathrm{agg}}=51 \mathrm{~nm}$ and $68 \mathrm{~nm}$ for silica loadings of $1 \%$ by weight and $10 \%$ by weight, respectively, to agree with SAXS fits on individual particles and the Guinier analysis. The fits adequately describe the scattering data (Fig. S4).

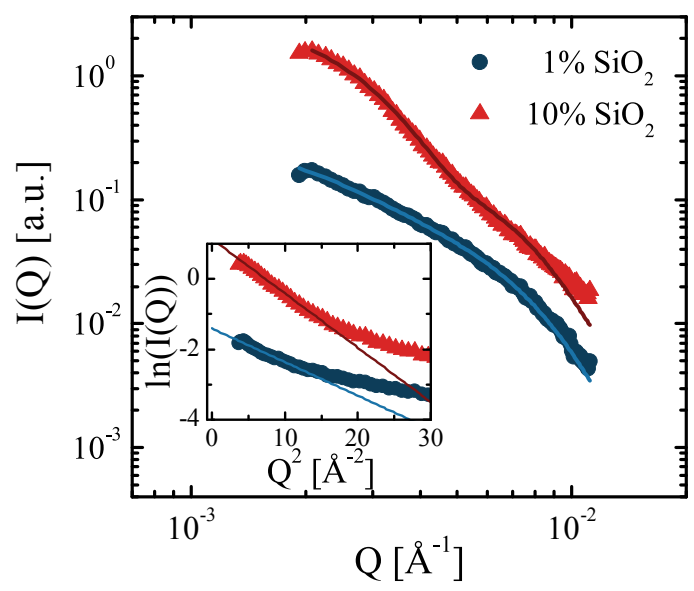

Figure S4: SAXS scattering intensity $I(Q)$ for solutions of $16.5 c^{*} \mathrm{PS} 1 \% \mathrm{SiO}_{2}$ (blue) and $18.5 c^{*} \mathrm{PS} 10 \% \mathrm{SiO}_{2}$ (red). Solid curves are fits to the sum of two spherical form factors. (Inset) Guinier plot of the same SAXS data. Solid lines indicate linear fits to low-Q data.

\section{Interparticle interactions: Depletion attraction and electrostatic repulsion}

To understand why the particles aggregate at high polymer loadings, we estimate the interparticle interactions. We extract the charge on the surface of a particle (Table SIII) from fits to structure factor obtained from the SAXS data. ${ }^{\mathrm{S} 4}$ The repulsive potential is estimated 
from the particle charge according to

$$
\Psi_{\text {rep }}=\pi \varepsilon_{0} \varepsilon \sigma^{2} \psi_{0}^{2} \exp [-\kappa(r-\sigma)] / r
$$

where $r$ is the distance between particles, $\sigma$ is the particle radius, $\psi_{0}$ is the surface potential, $\varepsilon$ is the solvent dielectric constant, $\varepsilon_{0}$ is the permittivity constant, and $\kappa$ is the inverse of the Debye length. The surface potential $\psi_{0}$ is related to the particle surface charge $z_{\mathrm{m}}$ by

$$
\psi_{0}=z_{\mathrm{m}} / \pi \varepsilon_{0} \varepsilon \sigma(2+\kappa \sigma) .{ }^{\mathrm{S} 4}
$$

The Debye length $\kappa^{-1}$ is estimated as

$$
\kappa^{-1}=\left(z_{\mathrm{m}}^{2} n / \varepsilon_{0} \varepsilon k_{\mathrm{B}} T\right)^{-1 / 2}
$$

where $n$ is the number density of particles in solution and $k_{\mathrm{B}}$ is the Boltzmann constant. ${ }^{\mathrm{S}}$ As a representative example, we calculate the repulsive potential for the $5.5 \mathrm{c}^{*} \mathrm{PS} 10 \% \mathrm{SiO}_{2}$ solution (Fig. S5).

Table SIII: Fitting parameters for the SAXS structure factors.

\begin{tabular}{|c|c|c|}
\hline Sample & Charge per particle $z_{\mathrm{m}}[\mathrm{e} \pm 0.5]$ & Volume fraction $\phi \pm 0.001$ \\
\hline $0 c^{*} \mathrm{PS} 1 \% \mathrm{SiO}_{2}$ & 28.0 & 0.004 \\
\hline $0 c^{*} \mathrm{PS} 3 \% \mathrm{SiO}_{2}$ & 67.3 & 0.014 \\
\hline $0 c^{*} \mathrm{PS} 10 \% \mathrm{SiO}_{2}$ & 66.3 & 0.069 \\
\hline $5.5 \mathrm{c}^{*} \mathrm{PS} 1 \% \mathrm{SiO}_{2}$ & 23.7 & 0.003 \\
\hline $5.5 \mathrm{c}^{*} \mathrm{PS} 3 \% \mathrm{SiO}_{2}$ & 38.0 & 0.006 \\
\hline $5.5 c^{*} \mathrm{PS} 10 \% \mathrm{SiO}_{2}$ & 79.0 & 0.024 \\
\hline $8 c^{*} \mathrm{PS} 1 \% \mathrm{SiO}_{2}$ & 19.2 & 0.002 \\
\hline $8 \mathrm{c}^{*} \mathrm{PS} 3 \% \mathrm{SiO}_{2}$ & 25.2 & 0.008 \\
\hline $8 c^{*} \mathrm{PS} 10 \% \mathrm{SiO}_{2}$ & 16.9 & 0.017 \\
\hline $12.5 c^{*} \mathrm{PS} 1 \% \mathrm{SiO}_{2}$ & 40.0 & 0.002 \\
\hline $12.5 c^{*} \mathrm{PS} 10 \% \mathrm{SiO}_{2}$ & 44.6 & 0.023 \\
\hline
\end{tabular}


In polymer solutions, the particles also experience an attractive potential due to depletion attractions. The attractive potential for large spheres in semidilute polymer solutions is expressed as

$$
\Psi_{\mathrm{dep}} / k_{\mathrm{B}} T=-\frac{R}{\xi^{3}}(\pi \xi-H)^{2}
$$

for $0 \leq H \leq \pi \xi$ and $\Psi_{\text {dep }}=0$ for $H>\pi \xi$, where $R$ is the particle radius, $\xi$ is polymer correlation length, and $h$ is the distance away from the particle surface. ${ }^{\mathrm{S} 6}$ We estimate the depletion interactions for the various polymer concentrations discussed in the main body of the text as shown in Fig. S5.

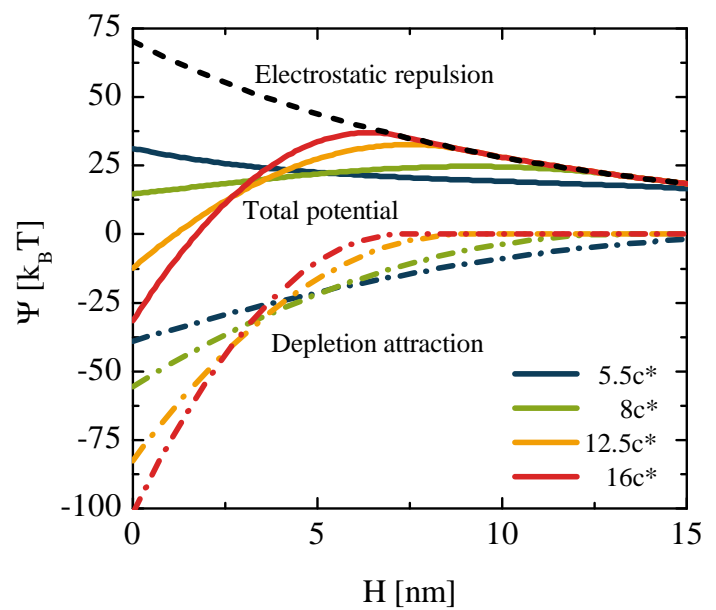

Figure S5: Estimates of interaction potentials $\Psi$ between silica nanoparticles in semidilute polystyrene solutions as a function of distance $H$ between particles. Dashed black line indicates representative electrostatic repulsions $\Psi_{\text {rep }}$ based on the parameters for the $5.5 \mathrm{c}^{*} \mathrm{PS}$, $10 \% \mathrm{SiO}_{2}$ solution. Dash-dot lines indicate depletion attractions $\Psi_{\text {dep }}$ at different polymer concentrations. Solid lines represent the sum of electrostatic repulsion and depletion attraction potentials.

Although both the repulsive and attractive interactions are rough estimates, they qualitatively predict the aggregation behavior that we measure. At contact $(H=0)$, the interparticle interactions are predicted to be fully repulsive $(\Psi>0)$ at low polymer concentrations but attractive $(\Psi<0)$ at high polymer concentrations. In solution, the depletion interactions overcome electrostatic repulsions in the highly concentrated $\left(\mathrm{c} / \mathrm{c}^{*}>15\right)$ polystyrene solutions, and the particles form aggregates of finite size. 


\section{SANS Fitting: Sphere and Lorentz}

Due to imperfect contrast matching, there is some scattering from the silica nanoparticles in the $8 c^{*}, 10 \% \mathrm{SiO}_{2}$ solution measured in SANS (Fig. 4(b) in main text). We fit the full set of scattering data to the sum of sphere and Lorentz form factors according to

$$
I_{\mathrm{coh}}(Q)=\frac{A}{1+(Q \xi)^{2}}+B\left[\frac{\sin (Q R)-Q R \cos (Q R)}{(Q R)^{3}}\right]^{2}
$$

where $A$ and $B$ are scaling factors. Specifying $R=24 \mathrm{~nm}$ as measured in SAXS on the same solution does not give a good fit at low $Q$ (Fig. S6). Instead, we let $R$ float during the fit and find $R=31 \mathrm{~nm}$. The difference in radii measured with SAXS and SANS likely derives from one of two sources: (1) a small layer of absorbed organic molecules on the silica ${ }^{\mathrm{S} 7}$ or (2) structuring of the solvent around the nanoparticles. ${ }^{\mathrm{S} 8}$ Both of these sources can be detected using neutrons but would be invisible to x-rays.

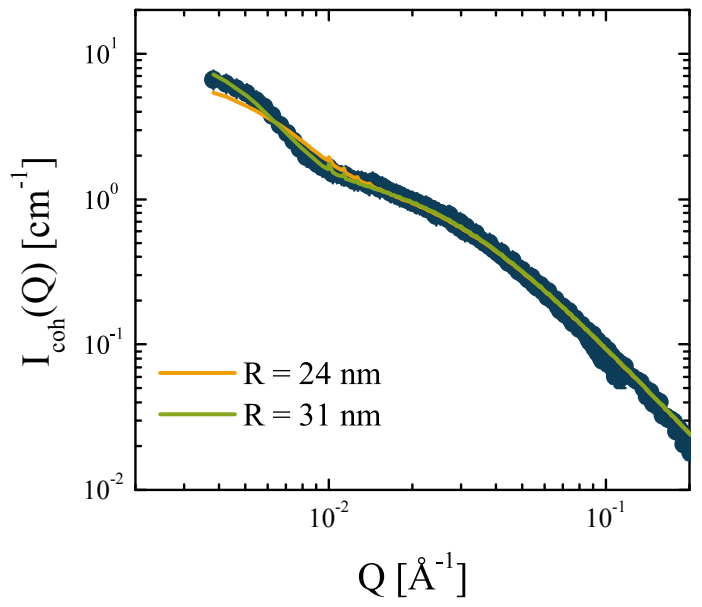

Figure S6: Scattering intensity for the $8 c^{*} \mathrm{PS} 10 \% \mathrm{SiO}_{2}$ solution in d5-2-butanone. Solid curves are fits to Eq. S7 with different radii. 


\section{XPCS Analysis}

We fit the XPCS autocorrelation curves for particle dynamics according to stretched exponentials with a fitting form of

$$
G_{2}(Q, t)=A+B \exp \left(-2(\Gamma t)^{\beta}\right)
$$

where $A \approx 1$ is the long time plateau, $B$ is the Siegert factor, $\beta$ is a stretching exponent, and $\Gamma$ is the relaxation rate. To check the quality of our fits, we perform two fitting schemes. In the first, we allow $\beta$ to vary at each $Q$. In the second fitting scheme, we fit the autocorrelation curves using a global value for $\beta$. Both fitting schemes give comparable values of $\beta$ and the corresponding relaxation rate $\Gamma$ as a function of $Q$ (Fig. S7). Thus, we fit the autocorrelation curves for each solution using global $\beta$ values.
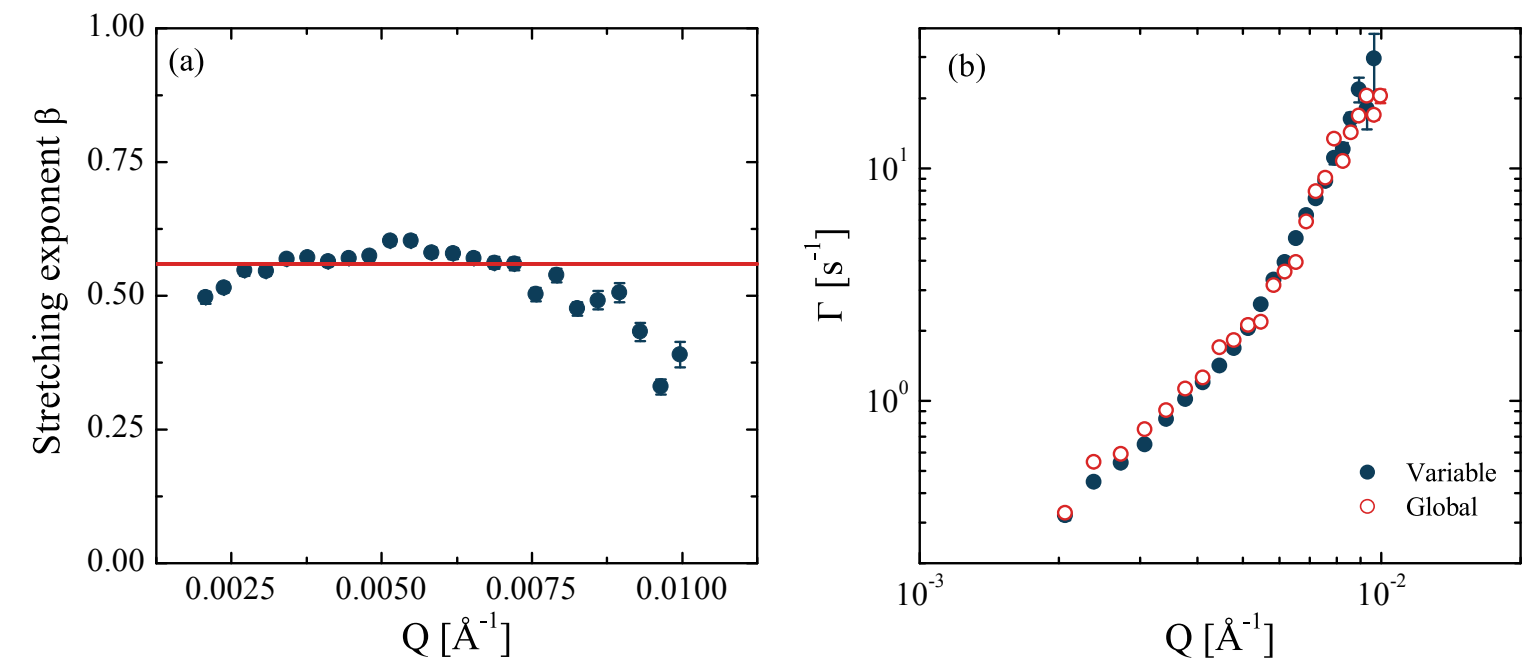

Figure S7: (a) Stretching exponent $\beta$ as a function of wavevector $Q$ for autocorrelation curves measured on the $8 \mathrm{c}^{*} \mathrm{PS} 10 \% \mathrm{SiO}_{2}$ solution. Solid line represents $\beta=0.56$ determined from global fitting scheme. (b) Relaxation rate $\Gamma$ as a function of $Q$ for the same solution. Closed symbols represent $\Gamma$ determined with variable $\beta$ fitting scheme. Open symbols represent $\Gamma$ determined with global $\beta$ fitting scheme.

We also check if a two-step relaxation model could fit our data using a fitting form of

$$
G_{2}(Q, t)=A+B\left[f \exp \left(-\Gamma_{1} t\right)+(1-f) \exp \left(-\Gamma_{2} t\right)\right]^{2}
$$


where $A \approx 1$ is the long time plateau, $B$ is the Siegert factor, $f$ is the relative contribution of the two relaxation steps to the overall decay of $G_{2}$, and $\Gamma_{1}$ and $\Gamma_{2}$ are the relaxation rates for the two steps. We fit each solution using global values of $A$ and $B$ and let $f$, $\Gamma_{1}$, and $\Gamma_{2}$ float for each $Q$ value. We compare the results of the two-step model with the stretched model for representative $Q$ values in Fig. S8. Both fitting forms result in residuals of comparable magnitude, but the double exponential fits introduce systematic oscillations to the residuals that are absent when using the stretched exponential model. Additionally, the stretched exponential model only requires four fitting parameters, three of which are globally fit, whereas the double exponential model requires five fitting parameters, only two of which can be globally fit. By using the stretched exponential fitting form, we achieve the same accuracy as the double exponential fitting form with less systematic deviation in the residuals and with fewer fitting parameters. Thus, we conclude that the stretched exponential fitting form is more appropriate for our data.

\section{Subdiffusive dynamics from stretched exponentials and scaling}

We want to verify that the fitting scheme used for the XPCS data does not introduce artifacts. The XPCS autocorrelation curves decay as stretched exponentials and the relaxation rates $\Gamma$ scale as a power law with $Q$. Together, the stretched exponentials and the relaxation rate scaling indicate subdiffusive dynamics. To verify that the stretching exponents $\beta$ and $Q$ scaling are independent of each other, we fit a representative set of autocorrelation curves with specified values of $\beta$. The relaxation rates $\Gamma$ for different $\beta$ values are all qualitatively similar although slightly different numerically (Fig. S9). Thus, we conclude that the $Q$ scaling and $\beta$ and independent of each other and are not a result of the fitting scheme. 

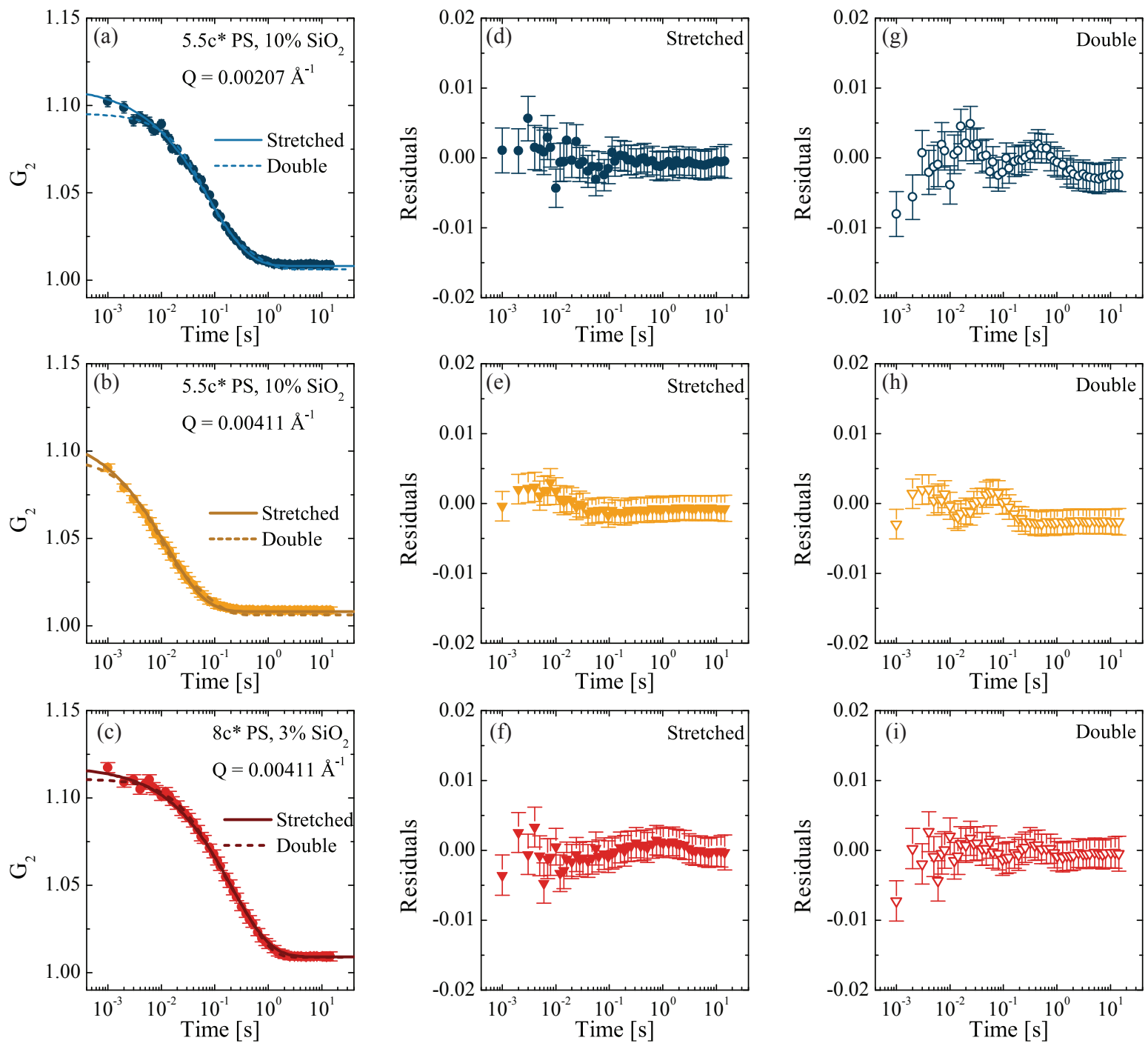

Figure S8: Representative comparisons of fits for intensity autocorrelation $G_{2}$ using stretched exponential (solid curves) (Eq. S8) and double exponential (dashed curves) (Eq. S9) fitting forms for the $5.5 \mathrm{c}^{*} \mathrm{PS} 10 \% \mathrm{SiO}_{2}$ solution at (a) $Q=0.00207 \AA^{-1}$ and (b) $Q=0.00411 \AA^{-1}$ and for the (c) $8 \mathrm{c}^{*}$ PS $10 \% \mathrm{SiO}_{2}$ solution at $Q=0.00411 \AA^{-1}$. Residuals from stretched (d, e, f) and double (g, h, i) exponential fitting forms for the same solutions and wavevectors. 

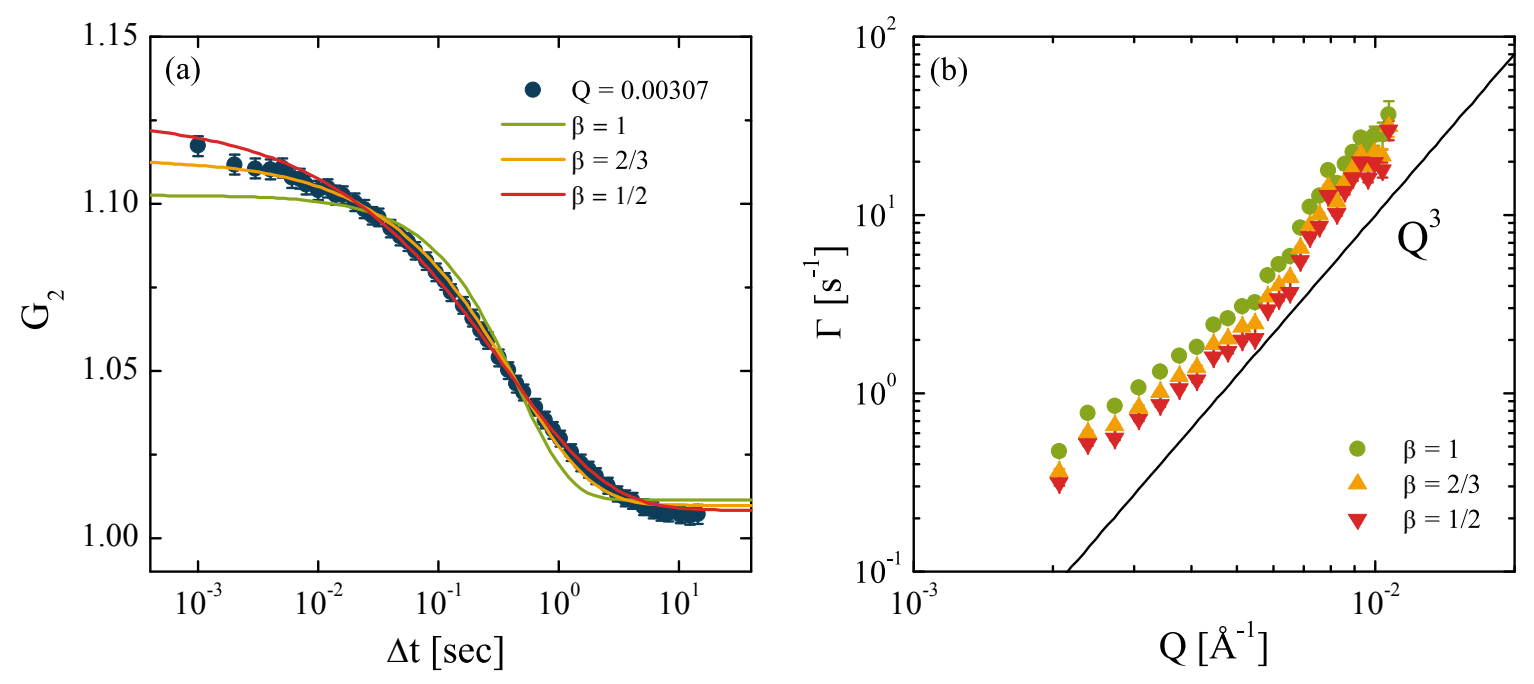

Figure S9: (a) Autocorrelation curve $G_{2}$ at $Q=0.00307 \AA^{-1}$ for particle dynamics in the $8 c^{*} \mathrm{PS} 10 \% \mathrm{SiO}_{2}$ solution. Solid lines represent best fits with various stretching exponents $\beta$. (b) Relaxation rates $\Gamma$ as a function of $Q$ for various values of $\beta$. Solid line indicates $Q^{3}$ scaling.

Disclaimer: The identification of any commercial product or trade name does not imply endorsement or recommendation by the National Institute of Standards and Technology.

\section{References}

[S1] Wagner, H. L. The Mark-Houwink-Sakurada Equation for the Viscosity of Atactic Polystyrene. J. Phys. Chem. Ref. Data 1985, 14, 1101-1106.

[S2] Lewis, M. E.; Nan, S.; Yunan, W.; Li, J.; Mays, J. W.; Hadjichristidis, N. Analysis of solution properties of polystyrene in 2-butanone in the framework of the hard-sphere model. Macromolecules 1991, 24, 6686-6689.

[S3] Park, S.; Chang, T.; Park, I. H. Static and hydrodynamic size of polystyrene coils in various solvents. Macromolecules 1991, 24, 5729-5731.

[S4] Hayter, J. B.; Penfold, J. An analytic structure factor for macroion solutions. Mol. Phys. 1981, 42, 109-118. 
[S5] Hiemenz, P. C.; Rajagopalan, R. Principles of colloid and surface chemistry, 3rd ed.; Marcel Dekker: New York, 1997.

[S6] Kleshchanok, D.; Tuinier, R.; Lang, P. R. Direct measurements of polymer-induced forces. J. Phys. Condens. Matter 2008, 20, 073101.

[S7] Fedeyko, J. M.; Vlachos, D. G.; Lobo, R. F. Formation and Structure of Self-Assembled Silica Nanoparticles in Basic Solutions of Organic and Inorganic Cations. Langmuir 2005, 21, 5197-5206.

[S8] Bellissent-Funel, M.-C.; Sridi-Dorbez, R.; Bosio, L. X-ray and neutron scattering studies of the structure of water at a hydrophobic surface. J. Chem. Phys. 1996, 104, 1002310029. 